\title{
EXPERIENCE RATING WITH CREDIBILITY ADJUSTMENT OF THE MANUAL PREMIUM
}

\author{
G. C. TAYLOR \\ Australia
}

Abstract

A common procedure for experience rating is to use Whitney's credibility formula with the manual premium per risk unit estimated by the observed average claim amount per risk unit. As pointed out by Whitney himself, this observed average also needs to be subjected to credibility adjustment. This suggestion is pursued in the paper and an experience rating procedure developed in which there are two stages:

(i) revision of the prior expected manual premium in accordance with the observed average claim amount per risk unit;

(ii) experience rating of the individual risk classes by the usual methods but using the manual premium obtained in stage (i).

Both stages of this procedure involve credibility indices, for which formulae are developed.

Conditions under which the "common procedure" referred to above is fairly reliable are found and it is seen, somewhat surprising$1 y$, that the procedure is often more valid than one expects on intuitive grounds. Care is necessary, however, when there are wide differences in size between different risk classes.

\section{INTRODUCTION}

Credibility theory, a science developed in the U.S.A. in the early part of this century, has in recent years gained increasing acceptance in Europe. To quote Derron. [3], "it is an entry into a new actuarial field, which-looking at the work published recently, above all in Italy and Switzerland-he (the European actuary) is apparently willing to tread on".

It is noteworthy that the theory has evolved in two rather 
distinct branches, referred to by Bailey [I, pp. 63-5] as the branches of "limited fluctuation credibility" and "greatest accuracy credibility". Basically, the distinction between them is that the first is used as a rate revising technique and the second as an experience rating technique. It is assumed that the reader is familiar with this distinction.

In this paper we wish to re-examine the question of experience rating, with particular reference to determination of the manual premium. Strictly, the manual premium, which is often determined by some fairly rough-and-ready means, should be subjected to some form of credibility adjustment itself. One procedure which suggests itself readily is to apply rate revision techniques to determine the manual premium and then to apply the usual experience rating techniques to determine the premium rates for the various risk classes. We shall consider some of the ostensible objections to such a procedure, but find eventually that the conjectured procedure is, in fact, quite workable.

Section 2 sets the scene for later calculations by giving a brief description of the standard model of greatest accuracy credibility. Section 3 discusses the problems of simultaneously determining the manual premium and premiums for individual risk classes, both by credibility methods. In Section 4 , the credibility model is extended in an attempt to overcome these problems, and the results emerging from it are examined in Section 5.

\section{A Review of the Greatest Accuracy Credibility Model}

Before proceeding on our more detailed analysis it will be useful for us to summarize the hypotheses and results of the existing greatest accuracy credibility model. The techniques involved were developed by Whitney [5] for experience rating purposes and have remained largely unchanged. Credibility theorists' insight into and facility in handling the formulae has, however, advanced considerably over the years, culminating in the elegant derivation by Buhlmann recently [2, pp. I02-3]. We shall tend to follow his methods.

We suppose that we are concerned with a collective of similar but not identical risks, and that this collective can be divided into a number of homogeneous sub-collectives having differing underlying (and unknown) true risk premiums. We call these subcollectives risk classes and characterize them by a variable $\theta$. 
Let

(i) $\mu$ be the expected claim amount per risk unit taken at random from the entire collective;

(ii) $\mu(\theta)$ be the expected claim amount per risk unit taken at random from risk class $\theta$ (we shall select our values of $\theta$ for the risk classes in such a way that $\mu(\theta)$ increases with $\theta$ );

(iii) $S(\theta)$ be the random variable, actual claim amount per risk unit in risk class $\theta$ during the year of experience.

We assume that

(iv) for risk units chosen at random from the collective, $\theta$ is subject to a d.f. $U(\theta)$;

(v) for given $\theta, S(\theta)$ is subject to a d.f. $G^{(\theta)}(S(\theta))$.

Now let

(vi) $V\left(\theta_{0}, S_{0}\right)$ be the probability that, for a risk unit chosen at random from the collective, $\theta \leq \theta_{0}$ and, for the $\theta$ possessed by this unit, $S(\theta) \leq S_{0}$; informally, $d V(\theta, S(\theta))=d U(\theta)$ $d_{S(\theta)} G^{(\theta)}(S(\theta))$.

The correct risk premium per risk unit in class $\theta$ is of course $\mu(\theta)$ but, since the data from this class will be insufficient for a sharp estimate of $\mu(\theta)$, we agree to charge a premium of $E[\mu(\theta) / S(\theta)]$ We then wish to find a best estimate of $E[\mu(\theta) / S(\theta)]$ of the form $a+b S(\theta)$ where $a$ and $b$ are independent of $\theta$-best in the sense that it minimizes

$$
E_{V}\left[\{E[\mu(\theta) / S(\theta)]-(a+b S(\theta))\}^{2}\right],
$$

where, in order to avoid ambiguity, we have subscripted the first expected value operator with a $V$ to indicate expected value with respect to the d.f. $V$.

It turns out that if we define

$$
\begin{aligned}
\sigma_{1}^{2} & =E_{V}\left[\{S(\theta)-\mu(\theta)\}^{2}\right], \\
\sigma_{2}^{2} & =E_{V}\left[\{\mu(\theta)-\mu\}^{2}\right], \\
Z & =\mathrm{I} /\left(\mathrm{I}+\frac{\sigma_{1}^{2}}{\sigma_{2}^{2}}\right),
\end{aligned}
$$$$
\text { and }
$$ 
then

$$
a=(\mathrm{I}-Z) \mu \text {, }
$$

and

$$
b=Z \text {. }
$$

The factor $Z$ is called the credibility of the year's experiences.

\section{The Problem with the Manual Premium}

Substituting equations (5) and (6) into our linearized estimate of $E[\mu(\theta) / S(\theta)]$, we see that the premium rate to be charged in risk class $\theta$ for the next year is:

$$
P(\theta)=(\mathrm{r}-Z) \mu+Z S(\theta) .
$$

If we assume that we can obtain a reasonable estimate (often a guess) of $Z$, then we can apply equation (7) if only we can estimate $\mu$. In practice, the usual procedure is to estimate $\mu$ by $P$, the average actual claim amount per risk unit in the entire collective in the year of experience, i.e.

$$
P=\int S(\theta) d U(\theta)
$$

Certainly, $P$ is an unbiassed estimator of $\mu$ as can easily be seen by taking expected values (w.r.t. $V$ ) of both sides of equation (8). However, the variance of $P$ depends strongly on the size of the collective and, in particular, if the collective is on the small side, then the difference between $P$ and $\mu$ may be rather large, and we may feel inclined to give some credibility to past experience. Whitney himself remarked on this point $[5$, p. 276]: "Another element that in theory may be taken account of is the varying credibility of the manual rate. The manual rate is established upon experience which in a majority of classifications is insufficient and which in many cases has been supplemented by judgement".

One suggestion of a solution to this problem was advanced in Section I where the possibility of dealing with experience rating in two distinct stages was noted. The first stage proposed involved temporarily disregarding the existence of the differing risk classes, and estimating $\mu$ by rate revision techniques, i.e. starting the experience year with some preconception of $\mu$ and using the experience data, viz. $P$, to modify it. This estimate of $\mu$ is then substituted in (7) to produce the second stage, the calculation of the 
premium rates for different risk classes by experience rating techniques.

Feasible though this suggestion is, there are, in fact, a couple of quite fundamental objections to it which call for consideration. Firstly, our decision in the first stage to overlook the existence of differing risk classes amounts to taking an unrealistic position, particularly since the assumptions of the second stage of our sequential procedure (i.e. the recognition of the differing risk classes) contradict those of the first. The effect of this factor is not clear from general considerations. Secondly, the second stage of the above procedure assumes that $\mu$ is a fixed parameter (see definition of $\mu$ in Section 2) and not a estimate of that parameter. Once again, the effect of this assumption is not clear, but one fact which is rather disquieting is that the two quantities substituted into equation (7), namely $S(\theta)$ and our estimate of $\mu$, are correlated since the estimate of $\mu$, being dependent on $P$, is a function of $S(\theta)$.

In the next two sections we shall find that, despite the apparent cogency of these objections, an experience rating procedure of the type suggested above does emerge in a natural way. Its emergence comes, however, only after a detailed analysis involving credibility indices perhaps a little different from those one would expect.

\section{Experience Rating with Credibility Adjustment of the Manual Premium}

In this section we wish to augment the model described in Section 2, incorporating the facility of rate revision of the manual premium. One means of achieving rate revision, as shown by Mayerson [4], is the adoption of Bayesian techniques. In our case this means using the statistic $\mathrm{P}$ to modify our preconceptions about $\mu$. In this spirit we add the following to the features (i) - (vi) of the model of Section 2:

(vii) $\mu$ has a prior distribution at the beginning of the year of experience. Let the d.f. be $F$;

(viii) for fixed $\mu$, each $\mu(\theta)$ is fixed;

(ix) for given $\mu, \theta$ and $\phi(\neq \theta), S(\theta)$ and $S(\phi)$ are stochastically independent;

(x) for given $\mu$ and $0, S(\theta)$ is subject to a d.f. $H_{\mu \theta}(S(\theta))$. 
Also $W\left(\mu_{0}, \theta_{0}, S\right)$ is the probability that the following three events all occur:

(a) $\mu \leq \mu_{0}$;

(b) $\theta \leq \theta_{0}$;

(c) $S$ is a function with domain $\boldsymbol{T}=\{$ values of $\theta$ in the collective $\}$, and $S(\phi) \leqslant S(\phi)$ for all $\phi \varepsilon T$.

Informally, $d W(\mu, \theta, \boldsymbol{S})=d F(\mu) d U(\theta) \Pi d_{\phi \varepsilon T} \boldsymbol{S}_{(\phi)} H_{\mu \phi}(\boldsymbol{S}(\phi))$;

We now wish to approximate $E[\mu(\theta) /\{S(\phi): \phi \varepsilon T\}]$ by $\alpha+\beta P+\gamma$ $(S(\theta)-P)$, where $\alpha, \beta, \gamma$ are independent of $\theta$, so that

$$
M=E_{W}\left[\{E[\mu(\theta) /\{S(\phi): \phi \varepsilon T\}]-(\alpha+\beta P+\gamma(S(\theta)-P))\}^{2}\right]
$$
is minimized.

It can be seen here that the experience rated premium $\alpha+\beta P+$ $\gamma(S(\theta)-P)$ consists of two components, viz. (i) $\alpha+\beta P$, the contribution of the rate-revised manual premium, and (ii) $\gamma(S(\theta)$ $P)$, the contribution of the experience-rated "deviation from standard".

To minimize (9), we need to find $\alpha, \beta^{\prime}(=\beta-\gamma)$ and $\gamma$ such that

$$
\begin{aligned}
& \partial M / \partial \alpha=0, \\
& \partial M / \partial \beta^{\prime}=0,
\end{aligned}
$$

$$
\text { and } \partial M / \partial \gamma=0 \text {, }
$$

simultaneously. Equation (Io) gives

$E_{W}\left[E[\mu(\theta) /\{S(\phi): \phi \varepsilon T\}]-\left(\alpha+\beta^{\prime} P+\gamma S(\theta)\right)\right]=0$.

i.e. $\quad m-\alpha-\beta^{\prime} m-\gamma m=0$,

i.e. $\quad \alpha=\left(\mathrm{I}-\beta^{\prime}-\gamma\right) m$,

where $m=E_{F}[\mu]$.

Using (13), we can put (9) in the form $M=E_{W}\left[\left\{(E[\mu(\theta) /\{S(\phi): \phi \varepsilon \boldsymbol{T}\}]-m)-\beta^{\prime}(P-m)-\gamma(S(\theta)-m)^{2}\right]\right.$, whereupon equations (II) and (I2) yield $E_{\boldsymbol{W}}\left[(P-m)\left\{(E[\mu(\theta) /\{S(\phi): \phi \varepsilon \boldsymbol{T}\}]-m)-\beta^{\prime}\left(P^{\prime}-m\right)-\gamma(S(0)-\right.\right.$ $m)\}]=0$, 
and

$$
\begin{gathered}
E_{W}\left[(S(\theta)-m)\{E[\mu(\theta) /\{S(\phi): \phi \varepsilon \boldsymbol{T}\}]-m)-\beta^{\prime}(P-m)-\gamma(S(\theta)-\right. \\
m)\}]=0 .
\end{gathered}
$$

That is,

$$
\operatorname{Cov}_{W}[\mu(\theta), P]-\beta^{\prime} \operatorname{Var}_{W}[P]-\gamma \operatorname{Cov}_{W}[S(\theta), P]=0,
$$

and

$$
\operatorname{Cov}_{W}[\mu(\theta), S(\theta)]-\beta^{\prime} \operatorname{Cov}_{W}[S(\theta), P]-\gamma \operatorname{Var}_{W}[S(\theta)]=0 .
$$

Solving (I4) and (I5) simultaneously:

$$
\begin{aligned}
& \beta^{\prime}=\frac{\operatorname{Var}_{W}[S(\theta)] \operatorname{Cov}_{W}[\mu(\theta), P]-\operatorname{Cov}_{W}[S(\theta), P] \operatorname{Cov}_{W}[\mu(\theta), S(\theta)]}{\operatorname{Var}_{W}[S(\theta)] \operatorname{Var}_{W}[P]-\left\{\operatorname{Cov}_{W}[S(\theta), P\}^{2}\right.}, \\
& \gamma=\frac{\operatorname{Var}_{W}[P] \operatorname{Cov}_{W}[\mu(\theta), S(\theta)]-\operatorname{Cov}_{W}[S(\theta), P] \operatorname{Cov}_{W}[\mu(\theta), P]}{\operatorname{Var}_{W}[S(\theta)] \operatorname{Var}_{W}[P]-\left\{\operatorname{Cov}_{W}[S(\theta), P]\right\}^{2}} .
\end{aligned}
$$

In order to simplify these equations, we define (as in equations (A.I) to (A.5) of the Appendix):

$$
\begin{aligned}
\sigma_{1}^{2}(\phi) & =E_{W}\left[\{S(\phi)-\mu(\phi)\}^{2} / \text { fixed } \phi\right] . \\
\sigma_{1}^{2} & =E_{W}\left[\{S(\theta)-\mu(\theta)\}^{2}\right]=\int \sigma_{1}^{2}(\phi) d U(\phi) . \\
\sigma_{2}^{2} & =E_{W}\left[\{\mu(\theta)-\mu\}^{2}\right] . \\
\sigma_{3}^{2} & =E_{W}\left[\{\mu-m\}^{2}\right] . \\
\sigma_{4}^{2} & =\int \sigma_{1}^{2}(\phi)[d U(\phi)]^{2} .
\end{aligned}
$$

As shown in the Appendix, all terms appearing in equations (I6) and (I7) can be constructed from the $\sigma_{i}^{2}(i=\mathrm{I}, 2,3,4)$. Proceeding thus, by means of equations (A. I2) to (A. I6) of the Appendix, we render (I6) and (I7) in the form

$$
\begin{gathered}
\beta^{\prime}=\frac{\sigma_{3}^{2}\left(\sigma_{1}^{2}-\sigma_{4}^{2}\right)-\sigma_{2}^{2} \sigma_{4}^{2}}{\left(\sigma_{3}^{2}+\sigma_{4}^{2}\right)\left(\sigma_{1}^{2}+\sigma_{2}^{2}-\sigma_{4}^{2}\right)}, \\
\gamma=\frac{\sigma_{2}^{2}}{\sigma_{1}^{2}+\sigma_{2}^{2}-\sigma_{4}^{2}} .
\end{gathered}
$$




\section{Examination of the results}

In the last section, we obtained an expression of the form $\alpha+\beta^{\prime} P+\gamma S(\theta)$ for $P(\theta)$, the premium rate applicable to risk class $\theta$, where $\alpha, \beta^{\prime}$ and $\gamma$ are given by equations (13), (23) and (24) respectively. The first observations that one would like to make on the extended theory which has been erected would be that it has as a subset the more restricted theory which was its starting point. An examination of our credibility formula in this light leads to some interesting considerations which are dealt with one by one below.

(i) Reducibility to the Bayesian Limited Fluctuation Credibility Formula

Suppose that there is only one risk class $\theta=\theta_{\circ}$, so that

$$
\begin{aligned}
U(\theta) & =0, \theta<\theta_{0} ; \\
& =\mathrm{I}, 0 \geqslant \theta_{0} ; \\
S(0) & =P, \\
\sigma_{1}^{2} & =\sigma_{4}^{2}=\sigma_{1}^{2}\left(\theta_{0}\right), \\
\sigma_{2}^{2} & =0
\end{aligned}
$$

Then our credibility formula becomes

$$
P(\theta)=\alpha+\beta^{\prime} P,
$$

and, according to the method followed in Section 4 , gives the Bayesian point-estimate of $\mu$. This is precisely the problem solved by Mayerson [4, pp. 95-7] in his Bayesian approach to limited fluctuation credibility.

Applying equations ( $\mathrm{r} 3$ ) and (23) to (27) and recalling (26) followed by (25) we find

$$
P(\theta)=\left(\mathrm{x}-\beta^{\prime}\right) m+\beta^{\prime} P,
$$

where

$$
\beta^{\prime}=\sigma_{3}^{2} /\left(\sigma_{1}^{2}+\sigma_{3}^{2}\right)
$$

This is just an alternative expression of the result obtained by Mayerson. 
(ii) Irreducibility to the Greatest Accuracy Credibility Formula

The greatest accuracy model of Section 2 was generalized to the model of Section 4 by the introduction of a prior distribution of $\mu$. If we now remove this distribution once again by setting

$$
\mu=m \text { and } \sigma_{3}^{2}=0,
$$

then equations (23) and (24) yield

$$
\beta^{\prime}=\gamma=\sigma_{2}^{2} /\left(\sigma_{1}^{2}+\sigma_{2}^{2}-\sigma_{4}^{2}\right) .
$$

Our credibility formula then becomes

$$
P(\theta)=\left(\mathrm{I}-2 \beta^{\prime}\right) m+\beta^{\prime} P+\beta^{\prime} S(\theta) .
$$

Now if we translate the standard greatest accuracy credibility formula, as given in Section 2, into the notation of this and the previous section, we obtain

$$
P(\theta)=\left(\mathrm{I}-\beta^{\prime \prime}\right) m+\beta^{\prime \prime} S(0),
$$

with

$$
\beta^{\prime \prime}=\sigma_{2}^{2} /\left(\sigma_{1}^{2}+\sigma_{3}^{2}\right)
$$

A comparison of (30) and (3I) with (28) and (29) shows not only that the coefficients $\beta^{\prime \prime}$ and $\beta^{\prime}$ differ in general, but also that the credibility formula arising out of Section 4 actually involves an extra lerm. This discrepancy is interesting and arises as follows. In the credibility model of Section 2 we obtained $P(\theta)$ as the best linear estimate of $E[\mu(\theta) / S(\theta)]$ based on $m$ and $S(\theta)$ only. Thus, we neglected the available information in respect of all the $S(\phi)$, $\phi \neq \theta$. In the model of Section 4 , this information is used since our formula for $P(\theta)$ involves $P$ in addition to $m$ and $S(\theta)$. In effect, $P$ gives us some information regarding the general amount of variation in our $S(\theta)$ 's. To see this more clearly, consider the case where $P$ differs widely from $m$. Now recall that $\mu$ is known (since $\sigma_{3}=0$ ), so that $P$ is indicating a large amount of random fluctuation in our $S(0)$ 's generally. Therefore, we should be a little sceptical of the more extreme values among the $S(\theta)$ 's. This is precisely what equation (29) accomplishes.

At first sight, it might appear that formula (29) is superior to (30) in that it makes use of additional information in the form of P. On the other hand, however, the superiority of (29) is most 
marked when $P$ differs from $m$ by a large amount, and in this case one might be inclined to question one of the basic assumptions of the model, that $m$ is known.

\section{(iii) A Tro-stage Credibility Formula}

Sections I and 3 have commented briefly on the possibility of applying rate revision techniques to the estimation of the manual premium, and then using experience rating techniques in conjunction with the revised manual premium. If this procedure were adopted, the revised manual premium would be

$$
P^{\prime}=\left(\mathrm{r}-Z_{P}\right) m+Z_{P} P,
$$

where $Z_{P}$ is the appropriate credibility index. The second stage would then yield

$$
P(\theta)=\left(\mathrm{I}-Z_{S}\right) P^{\prime}+Z_{S} S(\theta),
$$

where $Z_{S}$ is a second credibility index. If substitution of $(32)$ in (33) is to produce $P(\theta)=\left(I-\beta^{1}-\gamma\right)+\beta^{\prime} P+\gamma S(\theta)$, as required, then

$$
\begin{gathered}
Z_{p}=\frac{\beta^{\prime}}{\mathrm{I}-\gamma}=\frac{\sigma_{3}^{2}\left(\sigma_{1}^{2}-\sigma_{4}^{2}\right)-\sigma_{2}^{2} \sigma_{4}^{2}}{\left(\sigma_{3}^{2}+\sigma_{4}^{2}\right)\left(\sigma_{1}^{2}-\sigma_{4}^{2}\right)}=\left[\mathrm{I}-\frac{\sigma_{2}^{2}\left(\sigma_{4}^{2} / \sigma_{1}^{2}\right)}{\sigma_{3}^{2}\left(\mathrm{I}-\sigma_{4}^{2} / \sigma_{1}^{2}\right)}\right] / \\
{\left[\mathrm{I}+\frac{\sigma_{4}^{2}}{\sigma_{3}^{2}}\right],} \\
Z_{S}=\gamma=\frac{\sigma_{2}^{2}}{\sigma_{1}^{2}+\sigma_{2}^{2}-\sigma_{4}^{2}}=\mathrm{I} /\left(\mathrm{I}+\frac{\sigma_{1}^{2}}{\sigma_{2}^{2}}-\frac{\sigma_{4}^{2}}{\sigma_{2}^{2}}\right) .
\end{gathered}
$$

At this point it is worth noting that, because $\sigma_{1}^{2} \geqslant \sigma_{4}^{2}$, we have

$$
\mathrm{o} \geqslant Z_{P}, Z_{S} \geqslant \mathrm{I}
$$

so that it is reasonable to refer to $Z_{P}, Z_{S}$ as credibility indices. We might call $Z_{P}$ the collective credibility and $Z_{S}$ the risk-class credibility.

Thus we see that the two-stage credibility formula conjectured in earlier sections can be constructed with relatively simple credibility indices. We see also that these indices differ from those occurring in the ordinary Bayesian rate revision formula (see part (i) of this section) and the greatest accuracy credibility formula (see part (ii) ) mainly by the appearance of the term $\sigma_{4}^{2}$. 
If, however, $\sigma_{4}^{2} / \sigma_{1}^{2}, \sigma_{4}^{2} / \sigma_{2}^{2}$ and $\sigma_{4}^{2} / \sigma_{3}^{2}$ approximate to zero, then (34) and (35) yield

$$
\begin{gathered}
Z_{P} \cong \mathrm{I}, \\
Z_{S} \cong \mathrm{I} /\left(\mathrm{I}+\frac{\sigma_{1}^{2}}{\sigma_{2}^{2}}\right),
\end{gathered}
$$

in which case

$$
P(\theta)=\left(\mathrm{I}-Z_{S}\right) P+Z_{S} S(\theta),
$$

and we are back to our greatest accuracy credibility formula with $m$ replaced by $P$.

This is somewhat surprising because, if we review part (i) of this section, we observe that the necessary condition for $m$ to be disregarded in the Bayesian rate revision formula is

$$
\sigma_{1}^{2} / \sigma_{3}^{2} \cong 0
$$

However, $\sigma_{1}^{2} / \sigma_{3}^{2}$ plays a relatively minor role in determining the collective credibility in the extended model, a sufficient condition for disregarding $m$ here being

$$
\sigma_{4}^{2} / \sigma_{1}^{2}, \sigma_{4}^{2} / \sigma_{3}^{2} \cong 0 .
$$

On the other hand, it may be dangerous to ignore $m$ when there are wide differences in size between risk classes, particularly if a single risk class dominates the collective, for in this latter case $\sigma_{4}^{2} / \sigma_{1}^{2}$ would certainly not be small.

A useful approximation to equations (34), (35) can be obtained by considering the case in which the collective contains $N$ risk classes all of equal size and with

$$
\sigma_{1}^{2}(\phi)=\text { const. for all } \phi .
$$

Then

$$
\sigma_{4}^{2} / \sigma_{1}^{2}=\mathrm{I} / N
$$

which on substitution in (34) and (35), eliminates $\sigma_{4}^{2}$ and yields

$$
\begin{gathered}
Z_{p}=\left(\mathrm{I}-\frac{\mathrm{I}}{N-\mathrm{I}} \frac{\sigma_{2}^{2}}{\sigma_{3}^{2}}\right) /\left(\mathrm{I}+\frac{\mathrm{I}}{N} \frac{\sigma_{1}^{2}}{\sigma_{3}^{2}}\right), \\
Z_{s}=\mathrm{I} /\left(\mathrm{I}+\frac{N-\mathrm{I}}{N} \frac{\sigma_{1}^{2}}{\sigma_{2}^{2}}\right) .
\end{gathered}
$$




\section{Conclusion}

We have examined the problem of experience rating with credibility adjustment of the manual premium and found that it is possible to proceed by two stages-first, to revise the manual premium by more or less standard rate revision techniques, and second, using this adjusted manual premium, carry out experience rating in the usual fashion.

In carrying out this analysis we have found conditions under which the common procedure of adopting the standard greatest accuracy credibility formula with expected average claim amount for the collective replaced by observed average claim amount is a fairly valid one. These conditions are given in Section 5 (iii) leading to relations (36) and (37).

It is also found in the same section that this procedure may often be more valid than one expects. This is because, as is apparent from equations (38) and (39), the extended credibility tends to depend very much on the number of risk-classes in the collective as well as the number of risk units in them.

\section{ApPEndix}

The basic building blocks in the construction of the terms appearing in equations (I6) and (I 7$)$ are defined in equations (A. r) to (A. 8) below.

$$
\begin{aligned}
\sigma_{1}^{2}(\phi) & =E_{W}\left[\{S(\phi)-\mu(\phi)\}^{2} / \phi \text { fixed }\right] . \\
\sigma_{1}^{2} & =E_{W}\left[\{S(\theta)-\mu(\theta)\}^{2}\right]=\int \sigma_{1}^{2}(\phi) d U(\phi) . \\
\sigma_{2}^{2} & =E_{W}\left[\{\mu(\theta)-\mu\}^{2}\right] . \\
\sigma_{3}^{2} & =E_{W}\left[\{\mu-m\}^{2}\right] . \\
\sigma_{4}^{2} & =\int \sigma_{1}^{2}(\phi)[d U(\phi)]^{2} . \\
C_{S}(\phi, \psi) & =E_{W}[\{S(\phi)-\mu(\phi)\}\{S(\psi)-\mu(\psi)\} / \phi, \psi \text { fixed }] .
\end{aligned}
$$

Clearly, by assumption (ix) of our model,

$$
\begin{aligned}
C_{S}(\phi, \psi) & =o, \text { if } \phi \neq \psi, \\
& =\sigma_{1}^{2}(\phi) \text { if } \phi=\psi . \\
C_{\mu}(\phi, \psi) & =E_{W}[\{\mu(\phi)-\mu\}\{\mu(\psi)-\mu\} / \phi, \psi \text { fixed. }]
\end{aligned}
$$


Using this symbolism, we have

$\operatorname{Cov}_{W}[S(\phi), S(\psi) / \phi, \psi$ fixed $]=E_{W}[\{(S(\phi)-\mu(\phi))+(\mu(\phi)-\mu)$

$+(\mu-m)\}\{(S(\psi)-\mu(\psi))+(\mu(\psi)-\mu)+(\mu-m)\} / \phi, \psi$ fixed $]$

$=E_{W}[\{S(\phi)-\mu(\phi)\}\{S(\psi)-\mu(\psi)\} / \phi, \psi$ fixed $\}$

$+E_{W}[\{\mu(\phi)-\mu\}\{\mu(\psi)-\mu\} \phi, \psi$ fixed $\}+E_{W}\left[(\mu-m)^{2}\right]$

$=C_{S}(\phi, \psi)+C_{\mu}(\phi, \psi)+\sigma_{3}^{2}$.

Similarly,

$\operatorname{Cov}_{W}[S(\theta), S(\phi) / \phi$ fixed $]=E_{W}[\{S(\theta)-\mu(\theta)\}\{S(\phi)-\mu(\phi)\} / \phi$ fixed $]$

$+E_{W}[\{\mu(\theta)-\mu\}\{\mu(\phi)-\mu\} / \phi$ fixed $]+E_{W}\left[(\mu-m)^{2}\right]$

$=\int C_{S}(\theta, \phi) d U(\theta)+0+\sigma_{3}^{2}$

(using assumption (viii) of our model)

$$
=\sigma_{1}^{2}(\phi) d U(\phi)+\sigma_{3}^{2},
$$

by (A.7)

$\operatorname{Cov}_{W}[\mu(\theta), S(\phi) / \phi$ fixed $]=E_{W}[\{(\mu(\theta)-\mu)+(\mu-m)\}\{(S(\phi)-$ $\mu(\phi))+(\mu(\phi)-\mu)$

$+(\mu-m)\} / \phi$ fixed]

$=E_{W}[\{\mu(\theta)-\mu\}\{\mu(\phi)-\mu\} / \phi$ fixed $]-E_{W}\left[(\mu-m)^{2}\right]$

$=\sigma_{3}^{2}$,

by assumption (viii) of our model.

$\operatorname{Var}_{W}[S(\theta)]=E_{W}\left[\{(S(\theta)-\mu(\theta))+(\mu(\theta)-\mu)+(\mu-m)\}^{2}\right]$

$=E_{W}\left[\{S(\theta)-\mu(\theta)\}^{2}\right]+E_{W}\left[\{\mu(\theta)-\mu\}^{2}\right]+E_{W}\left[(\mu-m)^{2}\right]$

$=\sigma_{1}^{2}+\sigma_{2}^{2}+\sigma_{3}^{2}$.

$\operatorname{Cov}_{W}[\mu(\theta), S(\theta)]=E_{W}[\{(\mu(\theta)-\mu)+(\mu-m)\}\{(S(\theta)-\mu(\theta))$ $=E_{W}\left[\{\mu(\theta)-\mu\}^{2}\right]+E_{W}\left[(\mu-m)^{2}\right]$ $+(\mu(\theta)-\mu)+(\mu-m)\}]$

$=\sigma_{2}^{2}+\sigma_{3}^{2}$.

$\operatorname{Var}_{W}[P]=\operatorname{Var}_{W}\left[\int S(\phi) d U(\phi)\right]$

$=\iint \operatorname{Cov}_{W}[S(\phi), S(\psi) / \phi, \psi$ fixed $] d U(\phi) d U(\psi)$

$=\iint\left\{C_{S}(\phi, \psi)+C_{\mu}(\phi, \psi)+\sigma_{3}^{2}\right\} d U(\phi) d U(\psi)$

$=\int \sigma_{1}^{2}(\phi)[d U(\phi)]^{2}+\sigma_{3}^{2}$

(by (A.7) \& (A.8)) 


$$
=\sigma_{3}^{2}+\sigma_{4}^{2} .
$$

$\operatorname{Cov}_{W}[\mu(\theta), P]=\int \operatorname{Cov}_{W}[\mu(\theta), S(\phi) / \phi$ fixed $] d U(\phi)$

$$
=\sigma_{3}^{2}
$$

$\operatorname{Cov}_{w}[S(\theta), P]=\int \operatorname{Cov}_{W}[S(\theta), S(\phi) / \phi$ fixed $] d U(\phi)$,

$$
=\int\left\{\sigma_{1}^{2}(\phi) d U(\phi)+\sigma_{3}^{2}\right\} d U(\phi)
$$

$$
=\sigma_{3}^{2}+\sigma_{4}^{2} \text {. }
$$

\section{ReFERENCES}

[I] BaIley, Arthur L., Sampling Theory in Casualty Insurance, Proceedings Casualty Actuarial Society, Vol. 29, (1942), 50-79 and Vol. 3o (I943), $3 I-65$.

[2] Buhlmann, Hans, Mathematical Methods in Risk Theory, SpringerVerlag (I97I).

[3] Derron, Marcel, A Study in Credibility Betterment through Exclusion of the Largest Claims, Astin Bulletin, Vol. 4 Part I (1966), 39-48.

[4] Mayerson, Allen L., A Bayesian View of Credibility, Proceedings of the Casualty Actuarial Society, Vol. 5I (1964), 85-IO4.

[5] Whitney, Albert W., The Theory of Experience Rating, Proceedings of the Casualty Actuarial Society, Vol. 4 (1918), 274-292. 